\title{
Contrast-enhanced ultrasound for the assessment of focal nodular hyperplasia - results of a multicentre study
}

\author{
Roxana Şirli' ${ }^{1}$, Ioan Sporea ${ }^{1}$, Alina Popescu ${ }^{1}$, Mirela Dănilă ${ }^{1}$, Daniela Larisa Săndulescu ${ }^{2}$, \\ Adrian Săftoiu ${ }^{2}$, Tudor Moga ${ }^{1}$,Zeno Spârchez ${ }^{3}$, Cristina Cijevschi ${ }^{4}$, Cătălina Mihai ${ }^{4}$, \\ Simona Ioanițescu ${ }^{5}$, Dana Nedelcu ${ }^{6}$, Nicoleta Iacob $^{7}$, Grațian Miclăuș ${ }^{7}$, Ciprian Brisc ${ }^{8}$, \\ Radu Badea ${ }^{3}$
}

\begin{abstract}
${ }^{1}$ Department of Gastroenterology and Hepatology, "Victor Babeş" University of Medicine and Pharmacy Timişoara, ${ }^{2}$ Research Center of Gastroenterology and Hepatology, University of Medicine and Pharmacy Craiova, ${ }^{3}$ Regional Institute of Gastroenterology and Hepatology "Prof. Dr. Octavian Fodor", "Iuliu Hațieganu" University of Medicine and Pharmacy Cluj Napoca, ${ }^{4}$ Department of Gastroenterology, "Gr. T. Popa" University of Medicine and Pharmacy Iaşi, ${ }^{5}$ Center of Internal Medicine, Fundeni Clinical Institute, "Carol Davila" University of Medicine and Pharmacy, Bucharest, ${ }^{6}$ Ponderas and Neolife Hospitals, Bucharest, ${ }^{7}$ Department of Anatomy and Embryology, "Victor Babeş" University of Medicine and Pharmacy, Timişoara, ${ }^{8}$ Department of Gastroenterology, University of Oradea, Romania
\end{abstract}

\begin{abstract}
Aim: Contrast-enhanced ultrasound (CEUS) has become a relevant imaging method for the evaluation of focal liver lesions (FLL). The aim of this study was to evaluate the performance of CEUS for the assessment of focal nodular hyperplasia (FNH) in a large study group. Material and methods: We performed a multicentre prospective observational study, which included successive CEUS examinations from fourteen Romanian centres. CEUS examinations were performed in de novo FLL, using low mechanical index ultrasound, following an intravenous bolus of $2.4 \mathrm{ml}$ SonoVue. CEUS was considered conclusive for FNH if a typical pattern was present following contrast (rapid "spoke-wheel" enhancement during the arterial phase, hyperenhanced lesion during venous phase, hyper- or isoenhanced in the late phase). In all cases a reference method was available (contrast enhanced CT or MRI or biopsy). The trial was registered in clinicaltrials.gov (Identifier NCT01329458). Results: During the 6 years study, 2062 “de novo" FLL were evaluated by CEUS. From this cohort, 94/2062 (4.5\%) had a typical enhancing pattern for FNH as described in the EFSUMB guidelines. Contrast enhanced CT/MRI and biopsy diagnosed additional 15 FNH. From the 94 cases diagnosed as FNH by CEUS, in nine the final diagnosis was different (five of them adenomas). CEUS had $85 \%$ sensitivity, $99.5 \%$ specificity, $90.4 \%$ positive predictive value, $99.2 \%$ negative predictive value and $98.8 \%$ diagnostic accuracy for the diagnosis of FNH. Conclusions: CEUS is a sensitive and very specific method for the diagnosis of FNH.
\end{abstract}

Keywords: Contrast Enhanced Ultrasound; focal liver lesions; focal nodular hyperplasia; multicentre study

\section{Introduction}

Focal nodular hyperplasia (FNH) is the second most frequent benign liver tumour with a $3 \%$ estimat-

Received 01.11.2020 Accepted 25.02.2021

Med Ultrason

2021, Vol. 23, No 2, 140-146

Corresponding author: Ciprian Brisc, Assoc Prof

Department of Gastroenterology,

University of Oradea

65 Gheorghe Doja str, Oradea, România

E-mail: brisciprian@gmail.com

Phone: $+40-722591956$ ed prevalence, more frequent in women (female: male prevalence 4:1) [1]. It is not really a tumour but a regenerative nodule as a reaction to a vascular congenital abnormality [2,3]. Most frequently it is discovered by chance by conventional ultrasound in asymptomatic women younger than 50 , in $80-95 \%$ of cases as a solitary lesion, but in some cases multiple FNH can be found [4]. Considering their higher prevalence in women and the fact that they enlarge in women who use contraceptive drugs, FNH seems to be influenced by the hormone balance, even if hormones are not involved in their pathogenesis. 
From a pathological point of view, FNH include normal liver structures but with abnormal architecture. Typical for FNH is a fibrous scar (located in the centre, more rarely in the periphery), which includes an artery larger than usual, originating outside the nodule. This vascular disorder generates a regenerative nodule, which lacks the central terminal hepatic vein and has only capillarized sinusoids derived from the feeding artery [2]. This type of vascularization generates the typical aspect of $\mathrm{FNH}$ in contrast imaging.

On contrast enhanced ultrasound (CEUS), FNHs are hyper-vascular, appearing homogeneously hyperenhancing in the arterial phase, with very rapid and centrifugal fill-in. This "spoke-wheel" pattern is essential for differentiating FNHs from adenomas, from hyper-vascular malignant lesions, or from high-flow haemangiomas $[5,6]$. An eccentric feeding vessel may be seen in approximately $30 \%$ of cases. Usually, FNHs are hyperenhancing in the portal phase and hyper or isoenhancing in the late phase $[5,6]$. The central scar can appear as hypoenhancing in the late phase $[6,7]$. At contrast enhanced-CT, $\mathrm{FNH}$ is hyperdense with an isodense aspect during the portal-venous phase. A hyperdense central scar is commonly seen in the late phase [8]. On unenhanced MRI, FNHs appear as isointense on T1-weighted images and iso- to slightly hyperintense on T2-weighted images [9]. The MRI aspect of FNH following liver-specific contrast is of a hyperintense lesion in the initial phase with an isointense aspect in the delayed phase, the central scar being enhanced in the late phases [10].

FNHs are usually asymptomatic, being discovered during routine conventional US examination. Finding a focal liver lesion (FLL) can be highly stressful for the patient and thus all available methods should be performed to elucidate its nature. FNH's B-mode aspect is uncharacteristic; it can be isoechoic, slightly hyper- or hypoechoic, with the central scar visible in $70-80 \%$ of cases [11]. Baseline colour Doppler ultrasound can reveal the central vessel, originating from the centre to the periphery with a low resistance index and a high flow pattern [7].

In the latter years, CEUS has become a reliable imaging method for the evaluation of focal liver lesions. International and national guidelines define precise criteria for the diagnosis of FNH $[6,12]$. The aim of this study was to evaluate the performance of CEUS for the assessment of focal nodular hyperplasia in a large, prospective multicentre study, to collect useful information for the practitioner.

\section{Material and methods}

We performed a multicentre prospective observational study, during a 6-year period (April 2011-June 2017), which included successive CEUS examinations from eight university centres (14 individual departments). The trial was registered in clinicaltrials.gov (Identifier NCT01329458).

\section{Patients}

We included consecutive patients (older than 18 years), with newly discovered FLL during B-mode irrespective of the FLL size. In all patients, B-mode ultrasound was performed followed by CEUS. Also, in all patients a second line imaging method (contrast enhanced CT or MRI) or a biopsy were available, considered as the reference method. Most MRI imaging studies were performed using gadolinium based contrast media. Only in inconclusive cases hepato-specific contrast media was used. Demographic data, history of chronic hepatitis or of previous cancers, as well as data regarding the indication for CEUS were recorded.

The exclusion criteria were: contraindications for CEUS (acute myocardial infarction, class III/IV heart failure, rhythm disorders, pregnant women); patients who refused to participate; easy to diagnose FLL by conventional US, such as simple cysts or hydatid cysts, multiple lesions suggestive for liver metastases, patients with known FLL (hepatocellular carcinoma following percutaneous treatment, follow-up of patients with known metastasis or other FLLs, etc.); patients in whom biopsy or second line sectional imaging methods were unavailable.

A dedicated website was developed (http://study.umfcv.ro) for this study. Data were registered online for each individual patient.

Informed consent for CEUS was obtained from every patient. The study protocol was approved by the local Ethical Committee of each centre and was in accordance with the Helsinki Declaration of 1975.

\section{Conventional and contrast-enhanced studies}

In each patient, B-mode US and CEUS were performed with the same high-end ultrasound machine (different for each centre) able to perform low-mechanical index examinations. We documented the type of ultrasound machine, the name of the operator and the amount of contrast agent used. CEUS was performed by experts from each centre (levels II and III according to the EFSUMB classification), who were blinded to the CT/MRI or histology results.

Convex probes using a low mechanic index (0.090.11 ) were used. The US scan parameters (ie. focal zone, time gain compensation) were not changed during the CEUS study. One focus was placed below the lesion. We documented for each FLL the number, size, placement and US aspect on conventional B-mode ultrasound.

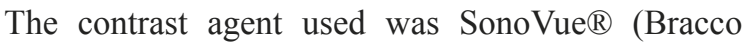
SpA, Milan, Italy), a perfluoro gas containing agent, as 


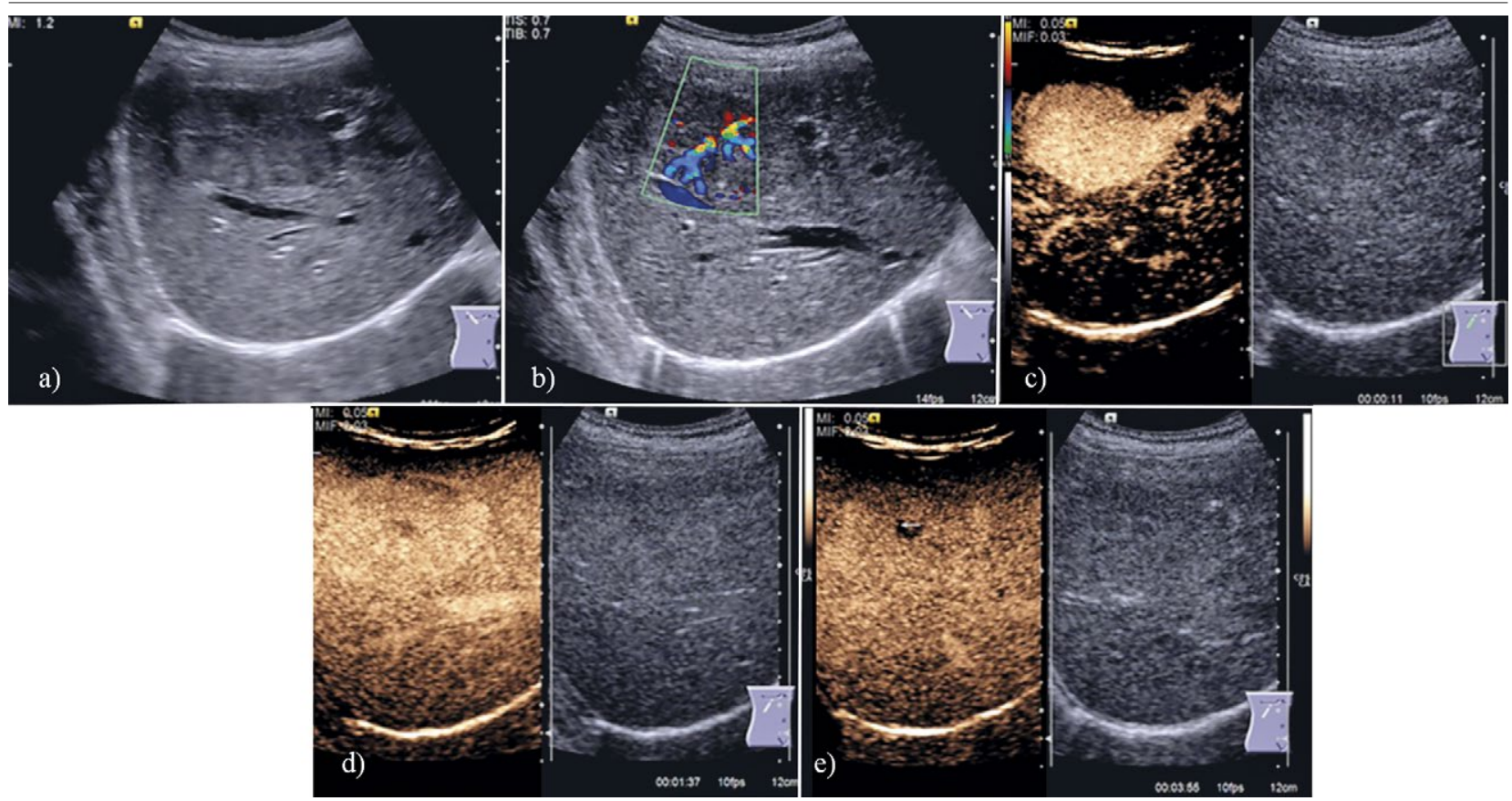

Fig 1. a) Slightly hypoechoic FLL in the right liver lobe. b) The FLL shows "spoke-wheel" pattern on Doppler US. c) CEUS, arterial phase - the lesion is completely hyperenhanced 11 seconds after bolus injection - excentric feeding artery also visible. d) CEUS, portal phase - the lesion is slightly hyperenhancing with visible central scar. e) CEUS, late phase - the lesion is iso/hyperenhancing with well visible central scar (arrow).

per standard protocol $[6,12]$. Lesions' enhancement patterns were studied in 3 phases: arterial (10-30 seconds following contrast bolus), portal (30-120 seconds) and late phase (>120 seconds) [6,12]. In each patient, the contrast study duration was at least 5 minutes after bolus injection. To document the study, four video files no longer than 30 seconds each were captured, containing conventional B-mode examination, the arterial phase, the portal phase and the late phase.

CEUS vascular patterns were defined by comparing the FLL's enhancement pattern to the surrounding liver parenchyma. We classified CEUS vascular patterns as: homogeneous hyperenhancement (the whole FLL showed homogeneous enhancement); heterogeneous hyperenhancement (mixed irregular areas of contrast enhancement were present); rim-like hyperenhancement (a peripheral hyperenhancement limited to less than 25\% of the tumour); iso-enhancement (the FLL enhanced the same as the adjacent parenchyma from the same depth); hypoenhancement (the lesion enhanced less than the adjacent parenchyma at the same depth); wash-out (hypoenhancement in the portal or late phases preceded by hyper or iso-enhancement in the arterial phase).

A CEUS diagnosis of FNH was established after the contrast study based on the patterns described in guidelines [6,12]: rapid "spoke-wheel" enhancement during the arterial phase, hyperenhanced lesion during the ve- nous phase, hyper or isoenhanced lesion in the late phase, sometimes with the visualization of a central hypoechoic scar (fig 1). A CEUS examination was considered conclusive if the FLL had a typical enhancement pattern according to national and international guidelines $[6,12]$ and inconclusive if not. The CEUS diagnosis was compared with the final diagnosis established based on all available imaging and clinical data: contrast enhanced $\mathrm{CT}$, and/or MRI, and/or histology.

\section{Statistical analysis}

Statistical analysis was performed using the GraphPad Prism program, version 7.02 (GraphPad Software, La Jolla, USA). We assessed the accuracy of CEUS for FNH characterization. The sensitivity (Se) was calculated as true positive cases divided by the total number of cases in which the disease was present; the specificity (Sp) was calculated as true negative cases divided by the total number of cases in which the disease was absent; the positive predictive value (PPV) was calculated as true positive cases divided by all CEUS positive cases; the negative predictive value (NPV) was calculated as true negative cases divided by all CEUS negative cases and accuracy was calculated as the sum of true positive and true negative cases divided by the total number of cases. We included in the statistical analysis all cases reported; we considered the inconclusive CEUS cases as wrongly diagnosed. 


\section{Results}

During the 6 years study, 2062 "de novo" FLL were evaluated by CEUS. Patients' characteristics are presented in Table I.

From this cohort, 94/2062 (4.5\%) had a typical enhancing pattern for FNH. Contrast enhanced CT/MRI and biopsy diagnosed additional 15 FNH (12 in cases labelled as inconclusive on CEUS and one each labelled by CEUS as haemangioma, adenoma and metastasis). From the 94 cases diagnosed as FNH by CEUS, in nine cases the final diagnosis was different (five of them adenomas, two of them hepatocellular carcinomas, one haemangioma and one focal fatty infiltration). Thus, the final diagnosis was FNH in 100 of the 2062 (4.8\%) cases. The characteristics of patients with $\mathrm{FNH}$ are presented in Table II.

The indication for CEUS was in most FNH cases an incidentally found FLL 98\% (98/100), in 1\% (1/100) case it was the evaluation of a patient with known oncologic disease and in 1\% (1/100) case it was an inconclusive CE-CT scan. At the moment of CEUS examination, none of the patients diagnosed by CEUS with FNH were known with underlying liver disease. However, two of them were later diagnosed with underlying cirrhosis and 2 with chronic hepatitis.

CE-MRI was the gold standard in 30\% (30/100) cases. In $6 \%(6 / 100)$ patients histology was available - one of them after surgery performed for HCC.

By comparing CEUS with the reference method (either CE-CT, CE-MRI or histology) CEUS had $85 \% \mathrm{Se}$, 99.5\% Sp, 90.4\% PPV, 99.2\% NPV and 98.8\% diagnostic accuracy for the diagnosis of $\mathrm{FNH}$.

\section{Discussion}

Focal liver lesions (FLLs) are frequently discovered during conventional B mode ultrasound (US) examination, either during a routine scan for various abdominal symptoms or during the follow-up of chronic liver disease or of oncologic patients. In most cases, only B mode US is not sufficient for a definite diagnosis. This is particularly true when FNH is involved, since its appearance is totally uncharacteristic, sometimes isoechoic, or slightly hyper- or hypoechoic, the most suggestive for the diagnosis being the central scar, which is visible only in $70-80 \%$ of cases $[8,10,11]$. Thus, a contrast imaging method or a histopathological exam are required to reach a final diagnosis. The time needed for scheduling, and the costs of contrast enhanced CT or contrast enhanced MRI are not negligible, the same is true regarding guided biopsy, which is also an invasive procedure. On the other
Table I. Patients' characteristics

\begin{tabular}{ll}
\hline Number of patients & $\mathbf{2 0 6 2}$ \\
\hline Mean age (years) & $52.4 \pm 7.5$ \\
Gender, women & $914(44.3)$ \\
Underlying chronic liver disease & $727(35.3 \%)$ \\
CEUS diagnosis & \\
$\quad$ Hepatocellular carcinomas & $685(33.2)$ \\
Hemangiomas & $452(21.9)$ \\
Metastases & $418(20.3)$ \\
Focal nodular hyperplasias & $94(4.5)$ \\
Regenerative nodules & $84(4.1)$ \\
Focal fatty infiltrations & $70(3.4)$ \\
Cholangiocarcinomas & $57(2.7)$ \\
Abscesses & $45(2.2)$ \\
Complex Cysts & $43(2.1)$ \\
Other benign lesions* & $37(1.8)$ \\
Adenomas & $32(1.6)$ \\
Fatty free areas & $26(1.3)$ \\
Other malignant lesions** & $19(0.9)$ \\
\hline
\end{tabular}

The results are expressed as number, number (\%) or mean \pm SD. * pseudolesion, scar area, angiomyolipoma, hamartoma; ** lymphoma, hemangiosarcoma, hepatic epithelioid hemangioendothelioma.

Table II. The characteristics of patients with focal nodular hyperplasia $(\mathrm{FNH})$

\begin{tabular}{ll}
\hline Number of patients with FNH & $\mathbf{1 0 0}$ \\
\hline Mean age & $40.4 \pm 13.7$ years \\
Gender, women & $75(75)$ \\
Underlying chronic liver disease & $4(4)$ \\
Size: & \\
$\quad \leq 35 \mathrm{~mm}$ & $39(39)$ \\
$\quad \geq 36 \mathrm{~mm}$ & $61(61)$ \\
\hline
\end{tabular}

The results are expressed as number (\%) or mean $\pm \mathrm{SD}$

hand, CEUS can be performed immediately after a conventional $\mathrm{B}$ mode US examination and thus a diagnosis can be established in minutes.

CEUS is a real-time imaging technique, which, similar to contrast enhanced CT or MRI, shows tissue perfusion, and is able to identify the type of FLL based on the enhancement pattern in arterial, portal and late vascular phases. The value of CEUS for the differential diagnosis of FLLs was proved in a German national multicentre study (DEGUM study) [13] and in a French national multicentre study (STIC study) [14], each including more than 1000 FLL and later in a Romanian multicentre study [15]. Meta-analyses demonstrated that CEUS accuracy is similar to contrast enhanced CT and MRI [16-18].

Other advantages of CEUS as compared with CT and MRI are the absence of radiation exposure (as in CT examination) and of side effects (no nephrotoxicity, no iodine exposure), and the lower costs $[19,20]$. Limitations of CEUS are linked to the limitations of US (poor acoustic window, uncooperative patients) and to the lesion's 
depth, since FLL located at more than $9 \mathrm{~cm}$ from the skin are difficult to examine due to attenuation $[6,12]$. However, if we compare the methods, we discover from the study of Bröker et al [21] that in a direct comparison between MRI with liver specific contrast and CEUS, MRI performed better than CEUS for the diagnosis of FNH and hepatocellular adenoma, CEUS having a sensitivity and specificity of $85 \%$ and $87 \%$, positive predictive value (PPV) of $79 \%$ and negative predictive value (NPV) of $90 \%$, while MRI reached $95 \%$ sensitivity, $95 \%$ specificity, 92\% PPV and 97\% NPV.

The first guidelines regarding the use of CEUS were issued in 2004 by the European Federation of Societies for Ultrasound in Medicine and Biology (EFSUMB) [22], which were revised in 2008 [23], in 2012 [6] and in 2020 [24], the last two developed in cooperation with the World Federation for Ultrasound in Medicine and Biology (WFUMB), thus with universal validity. Regarding the dose of SonoVue, guidelines recommend the use of $2.4 \mathrm{ml}(1 / 2 \mathrm{vial})$ for the characterization of FLL, even if in the last years, with the development of more performant US machines, doses as low as $1.2 \mathrm{ml}$ are frequently used [25]. A too high dose can lead to apparition of artifacts (shadowing, over-enhancement of small structures and signal saturation, as well as "drowning" of small lesions in the signal of adjacent structures). A too low dose can lead to an insufficient quantity of microbubbles in the late phases, so that the wash-out cannot be observed. However, if the dose is not optimal, the injection can be repeated, with a higher or lower dose, as needed [25]. Furthermore, to better evaluate the wash-out, insonation can be avoided in the arterial and portal phase of the reinjection, especially useful for diagnosis of hepatocellular carcinoma [26].

According to published data, CEUS is an accurate method to characterise FNH. In the DEGUM study the accuracy for FNH was $95.5 \%$ [27], with $57.1 \%$ Se and $99.3 \% \mathrm{Sp}$. In the STIC study, CEUS had $82.5 \%$ Se and $94.3 \% \mathrm{Sp}$ for the diagnosis of FNH [14]. The calculated pooled sensitivity for FNH was $88 \%$ in the FriedrichRust et al meta-analysis [18] and the Sp can go as high as $100 \%[14,28]$. In another study that evaluated the accuracy of CEUS performed by two operators for the diagnosis of 85 biopsy proven FNH, CEUS had $80.9 \% \mathrm{Se}$, 95.7\% Sp, 95.0\% PPV, 83.3\% NPV and $88.3 \%$ accuracy for operator one and $78.7 \% \mathrm{Se}, 93.6 \% \mathrm{Sp}, 92.5 \% \mathrm{PPV}$, $81.5 \%$ NPV and $86.2 \%$ accuracy for operator two. This study concluded that CEUS performed better than contrast enhanced CT for characterizing dynamic centrifugal filling or the "spoke-wheel" sign in small lesions [29].

In our study, the performance of CEUS to diagnose FNH was very good with excellent Sp (99.5\%) and ac- curacy $(98.8 \%)$ and a slightly lower Se (85\%). Regarding the misdiagnosed cases, most confusions were made with adenomas. Five cases diagnosed by CEUS as FNH were in fact adenomas and one case diagnosed by CEUS as adenoma was in fact FNH. The confusion is probably understandable since both types of FLL show rapid hyperenhancement in the arterial phase, but in adenoma the fill-in occurs initially at the periphery with very rapid centripetal filling, as opposed to the centrifuge filling seen in FNH.

Arterial phase can be better evaluated using parametric arrival time (PAT), a post processing modality of evaluation. In the portal phase, adenoma usually becomes isoechoic or, more rarely, remains slightly hyperechoic. Sometimes, in adenomas the washout occurs in the late phase, thus being false positive for malignancy $[6,12]$. The imaging diagnosis of adenoma is very difficult and often guided biopsy is needed. In the DEGUM study, CEUS correctly diagnosed $57.9 \%$ of the adenomas [13]. Computer-assisted diagnosis can also be used to improve the accuracy of differential diagnosis by CEUS between FNH and adenoma [30].

In the Roche et al study [31] were included $43 \mathrm{FNH}$ and 20 adenomas, most lesions diagnosed based on the histopathological exam. The conclusion of this study was that CEUS had excellent Sp for diagnosing FNH $(100 \%)$, but the Se varies according to the lesion's size: $93 \%$ for lesions $\leq 35 \mathrm{~mm}$ and $7.7 \%$ for lesions $>35 \mathrm{~mm}$, the overall Se being $67.4 \%$. The authors explained this observation by the fact that larger FNH have an increased vascular supply and several feeding arteries and thus, the typical spoke-wheel pattern is not visible. The same observation, that FNHs with signs of centrifugal filling were smaller than those without the sign $(3.1 \pm 1.5 \mathrm{~cm}$ vs. $5.2 \pm 3.2 \mathrm{~cm}, \mathrm{p}=0.000$ ) was made in the study by Wang et al [29]. In our study, the five FNH misdiagnosed as adenomas were $2.5 \mathrm{~cm}, 6 \mathrm{~cm}, 6 \mathrm{~cm}, 3 \mathrm{~cm}, 7.5$ and $3.2 \mathrm{~cm}$ in diameter, respectively.

Even if both adenoma and FNH are benign lesions, it is important to be differentiated since in adenoma there is a risk of rupture (and subsequent bleeding) or malignant transformation.

MRI with liver specific-contrast seems to outperform CEUS in differentiating FNH form hepatocellular adenoma. In a study by Grazioli et al, MRI had $96.9 \%$ Se, $100 \% \mathrm{Sp}, 100 \% \mathrm{PPV}, 96.4 \% \mathrm{NPV}$, and $98.3 \%$ overall accuracy [32].

Two FLL diagnosed by CEUS as FNH proved to be hepatocellular carcinomas (HCCs). Both types of lesions have arterial hyperenhancement but in HCC there is a chaotic pattern $[6,12]$. Usually, HCCs show mild, late, or very late washout, correlated with the differentiation of 
the tumour $[33,34]$. Well-differentiated HCCs show very late or no washout, thus the CEUS examination should take at least 4-5 minutes [35]. Thus, in well-differentiated HCCs, the CEUS aspect can be similar to that of FNH but there is a very important factor that should be taken into consideration: up to $90 \%$ of HCCs occur on a background of chronic liver disease with severe fibrosis and cirrhosis [36]. US based elastography, available in most US machines able to perform CEUS, should be used to rule in or rule out the presence of cirrhosis in a point of care US evaluation [37]. Thus, it is important to keep in mind that a new lesion on a cirrhotic liver, hyperenhancing in the arterial phase on CEUS, is probably HCC [35,38]. Computer assisted diagnosis [39] as well as parametric imaging could be used to improve the differential diagnosis by CEUS between FNH and HCC, the mean transit time being significantly longer in $\mathrm{FNH}$ than in HCC [40].

Both HCCs misdiagnosed as FNH were in patients with incidentally discovered FLL, who were not known with chronic liver disease, but in whom, starting from the HCC, compensated liver cirrhosis was diagnosed.

A limitation of or study is that the pathological exam was available only in a small number of cases (only 7 of the $100 \mathrm{FNH}$ ), but an advantage is the large number included and the prospective design of the study.

In conclusion, CEUS is an accurate method to diagnose $\mathrm{FNH}$, the main difficulties occurring in differentiating FNH from adenomas, especially in large lesions.

\section{Acknowledgements}

Part of the research published in this paper was made with support from the grant awarded by the "Victor Babeş" University of Medicine and Pharmacy Timişoara, in PROGRAMUL III - C2 - PCFI - 2015/2016.

The authors wish to acknowledge the help provided by the following colleagues: Dorina Pestroiu Calescu, Lucian Ciobâca, Liana Gheorghe, Mihai Socaciu, Alina Martie, Siegfried Christian Ivasc, Attila Tamas, Costin Theodor Streba, Mihaela Iordache, Iulia Simionov, Mariana Jinga, Adrian Anghel, Silviu Marcel Stanciu, Daniel Stoicescu, Eugen Dumitru, Corina Pietrăreanu, Daniela Bartoş, Roberta Mânzat Saplacan, Iuliana Pârvulescu, Roxana Vădan, Gabriela Smira and Liliana Tuţă

\section{References}

1. Karhunen PJ. Benign hepatic tumours and tumour like conditions in men. J Clin Pathol 1986;39:183-188.

2. Fukukura Y, Nakashima O, Kusaba A, Kage M, Kojiro M. Angioarchitecture and blood circulation in focal nodular hyperplasia of the liver. J Hepatol 1998;29:470-475.
3. Gaiani S, Celli N, Piscaglia F, et al. Usefulness of contrastenhanced perfusional sonography in the assessment of hepatocellular carcinoma hypervascular at spiral computed tomography. J Hepatol 2004;41:421-426.

4. Wanless IR, Albrecht S, Bilbao J, et al. Multiple focal nodular hyperplasia of the liver associated with vascular malformations of various organs and neoplasia of the brain: a new syndrome. Mod Pathol 1989;2:456-462.

5. Dietrich CF, Schuessler G, Trojan J, Fellbaum C, Ignee A. Differentiation of focal nodular hyperplasia and hepatocellular adenoma by contrast-enhanced ultrasound. Br J Radiol 2005;78:704-707.

6. Claudon M, Dietrich CF, Choi BI, et al. Guidelines and good clinical practice recommendations for Contrast Enhanced Ultrasound (CEUS) in the liver - update 2012: A WFUMB-EFSUMB initiative in cooperation with representatives of AFSUMB, AIUM, ASUM, FLAUS and ICUS. Ultrasound Med Biol 2013;39:187-210.

7. Piscaglia F, Lencioni R, Sagrini E, et al. Characterization of focal liver lesions with contrast-enhanced ultrasound. U1trasound Med Biol 2010;36:531-550.

8. Carlson SK, Johnson CD, Bender CE, Welch TJ. CT of focal nodular hyperplasia of the liver. AJR Am J Roentgenol 2000;174:705-712.

9. Ricci P, Laghi A, Cantisani V, et al. Contrast-enhanced sonography with SonoVue: enhancement patterns of benign focal liver lesions and correlation with dynamic gadobenate dimeglumine-enhanced MRI. AJR Am J Roentgenol 2005; 184:821-827.

10. Irie H, Honda H, Kaneko K, et al. MR imaging of focal nodular hyperplasia of the liver: value of contrast-enhanced dynamic study. Radiat Med 1997;15:29-35.

11. D'Onofrio M, Crosara S, De Robertis R, Canestrini S, Mucelli RP. Contrast-Enhanced Ultrasound of Focal Liver Lesions. AJR Am J Roentgenol 2015;205:W56-W66.

12. Sporea I, Badea R, Brisc C, et al. Romanian National Guidelines on Contrast Enhanced Ultrasound in clinical practice. Med Ultrason 2017;19:401-415.

13. Strobel D, Seitz K, Blank W, et al. Contrast-enhanced ultrasound for the characterization of focal liver lesions--diagnostic accuracy in clinical practice (DEGUM multicenter trial). Ultraschall Med 2008;29:499-505.

14. Tranquart F LGA, Correas JM, Ladam Marcus V, et al. Role of contrast-enhanced ultrasound in the blinded assessment of focal lesions in comparison with MDCT and CEMRI: Results from a multicentre clinical trial. Eur J Cancer Suppl 2008;.6:9-15.

15. Sporea I, Badea R, Popescu A, et al. Contrast-enhanced ultrasound (CEUS) for the evaluation of focal liver lesions - a prospective multicenter study of its usefulness in clinical practice. Ultraschall Med 2014;35:259-266.

16. Xie L, Guang Y, Ding H, Cai A, Huang Y. Diagnostic value of contrast-enhanced ultrasound, computed tomography and magnetic resonance imaging for focal liver lesions: a meta-analysis. Ultrasound Med Biol 2011;37:854-861.

17. Guang Y, Xie L, Ding H, Cai A, Huang Y. Diagnosis value of focal liver lesions with SonoVue(R)-enhanced ultra- 
sound compared with contrast-enhanced computed tomography and contrast-enhanced MRI: a meta-analysis. J Cancer Res Clin Oncol 2011;137:1595-1605.

18. Friedrich-Rust M, Klopffleisch T, Nierhoff J, et al. Contrast-Enhanced Ultrasound for the differentiation of benign and malignant focal liver lesions: a meta-analysis. Liver Int 2013;33:739-755.

19. Giesel FL, Delorme S, Sibbel R, Kauczor HU, Krix M. Contrast-enhanced ultrasound for the characterization of incidental liver lesions - an economical evaluation in comparison with multi-phase computed tomography. Ultraschall Med 2009;30:259-268.

20. Sirli R, Sporea I, Martie A, Popescu A, Danila M. Contrast enhanced ultrasound in focal liver lesions--a cost efficiency study. Med Ultrason 2010;12:280-285.

21. Broker MEE, Taimr P, de Vries M, et al. Performance of Contrast-Enhanced Sonography Versus MRI With a Liver-Specific Contrast Agent for Diagnosis of Hepatocellular Adenoma and Focal Nodular Hyperplasia. AJR Am J Roentgenol 2020;214:81-89.

22. Albrecht T, Blomley M, Bolondi L, et al. Guidelines for the use of contrast agents in ultrasound. January 2004. U1traschall Med 2004;25:249-256.

23. Claudon M, Cosgrove D, Albrecht T, et al. Guidelines and good clinical practice recommendations for contrast enhanced ultrasound (CEUS) - update 2008. Ultraschall Med 2008;29:28-44.

24. Dietrich CF, Nolsoe CP, Barr RG, et al. Guidelines and Good Clinical Practice Recommendations for Contrast Enhanced Ultrasound (CEUS) in the Liver - Update 2020 WFUMB in Cooperation with EFSUMB, AFSUMB, AIUM, and FLAUS. Ultraschall Med 2020;41:562-585.

25. Dietrich CF, Averkiou M, Nielsen MB, et al. How to perform Contrast-Enhanced Ultrasound (CEUS). Ultrasound Int Open 2018;4:E2-E15.

26. Dietrich CF, Ignee A, Greis C, Cui XW, Schreiber-Dietrich DG, Hocke M. Artifacts and pitfalls in contrast-enhanced ultrasound of the liver. Ultraschall Med 2014;35:108-125.

27. Seitz K, Strobel D, Bernatik T, et al. Contrast-Enhanced Ultrasound (CEUS) for the characterization of focal liver lesions - prospective comparison in clinical practice: CEUS vs. CT (DEGUM multicenter trial). Parts of this manuscript were presented at the Ultrasound Dreilandertreffen 2008, Davos. Ultraschall Med 2009;30:383-389.

28. Trillaud H, Bruel JM, Valette PJ, et al. Characterization of focal liver lesions with SonoVue-enhanced sonography: international multicenter-study in comparison to CT and MRI. World J Gastroenterol 2009; 15:3748-3756.

29. Wang W, Chen LD, Lu MD, et al. Contrast-enhanced ultrasound features of histologically proven focal nodular hy- perplasia: diagnostic performance compared with contrastenhanced CT. Eur Radiol 2013;23:2546-2554.

30. Denis de Senneville B, Frulio N, Laumonier H, Salut C, Lafitte L, Trillaud H. Liver contrast-enhanced sonography: computer-assisted differentiation between focal nodular hyperplasia and inflammatory hepatocellular adenoma by reference to microbubble transport patterns. Eur Radiol 2020;30:2995-3003.

31. Roche V, Pigneur F, Tselikas L, et al. Differentiation of focal nodular hyperplasia from hepatocellular adenomas with low-mechanical-index contrast-enhanced sonography (CEUS): effect of size on diagnostic confidence. Eur Radiol 2015;25:186-195.

32. Grazioli L, Morana G, Kirchin MA, Schneider G. Accurate differentiation of focal nodular hyperplasia from hepatic adenoma at gadobenate dimeglumine-enhanced MR imaging: prospective study. Radiology 2005;236:166177.

33. Boozari B, Soudah B, Rifai K, et al. Grading of hypervascular hepatocellular carcinoma using late phase of contrast enhanced sonography - a prospective study. Dig Liver Dis 2011;43:484-490.

34. Liu GJ, Xu HX, Lu MD, et al. Correlation between enhancement pattern of hepatocellular carcinoma on realtime contrast-enhanced ultrasound and tumour cellular differentiation on histopathology. Br J Radiol 2007;80:321330.

35. Bolondi L, Gaiani S, Celli N, et al. Characterization of small nodules in cirrhosis by assessment of vascularity: the problem of hypovascular hepatocellular carcinoma. Hepatology 2005;42:27-34.

36. Sherman M. Hepatocellular carcinoma: epidemiology, surveillance, and diagnosis. Semin Liver Dis 2010;30:316.

37. Ferraioli G, Wong VW, Castera L, et al. Liver Ultrasound Elastography: An Update to the World Federation for U1trasound in Medicine and Biology Guidelines and Recommendations. Ultrasound Med Biol 2018;44:2419-2440.

38. Jang HJ, Kim TK, Wilson SR. Small nodules $(1-2 \mathrm{~cm})$ in liver cirrhosis: characterization with contrast-enhanced ultrasound. Eur J Radiol 2009;72:418-424.

39. Huang Q, Pan F, Li W, et al. Differential Diagnosis of Atypical Hepatocellular Carcinoma in Contrast-Enhanced Ultrasound Using Spatio-Temporal Diagnostic Semantics. IEEE J Biomed Health Inform 2020;24:2860-2869.

40. Zheng SG, Xu HX, Liu LN, et al. Parametric imaging with contrast-enhanced ultrasound: usefulness for characterization of dynamic effects of microvascularization for hepatocellular carcinoma and focal nodular hyperplasia. Clin Hemorheol Microcirc 2013;55:375-389. 